\title{
Dietary Ziziphus jujuba Fruit Influence on Aberrant Crypt Formation and Blood Cells in Colitis-Associated Colorectal Cancer Mice
}

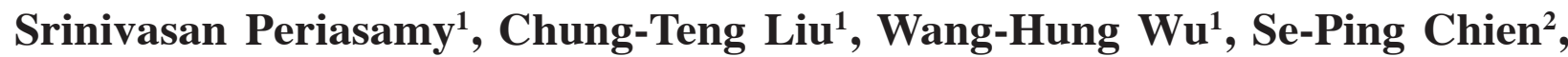 \\ Ming-Yie Liu ${ }^{1 *}$
}

\begin{abstract}
Ziziphus jujuba (ZJ) fruit is rich in bioactive functional components such as polysaccharides, triterpenoid acid, flavonoids and oleamide. It has been commonly used in the treatment of various diseases including diabetes, digestive disorders, diarrhea, skin infections, liver and urinary diseases. However, its dietary effect on chemoprevention of colon cancer has never been studied. The present study was to evaluate the protective effects of dietary $\mathrm{ZJ}$ on colitis-associated colon carcinogenesis in azoxymethane (AOM)-dextran sodium sulphate (DSS)-treated mice. AOM was injected (10 mg/kg b.wt., i.p.) and three cycles of $2 \%$ DSS in drinking water for 7 days with 14 days of normal drinking water in-between was administered to induce colitis-associated colon cancer. ZJ fruit was supplemented in feed as 5 and $10 \%$. Dietary ZJ significantly attenuated aberrant crypt foci (ACF) formation thereby decreasing the progression of hyperplasia to dysplasia. In addition, it significantly reduced circulating white blood cells, lymphocytes, neutrophils, monocytes, eosinophils, basophils and platelets compared to colon cancer mice. We conclude that $\mathrm{ZJ}$ supplementation delayed the progression of colon cancer from hyperplasia to dysplasia and ultimately adenocarcinoma and cancer. In addition, it decreased circulating tumor-related leucocytes, main regulators of cancer inflammation. Therefore, dietary consumption of $\mathrm{ZJ}$ fruit attenuated the formation of $\mathrm{ACF}$ and delayed the progression of colon cancer.
\end{abstract}

Keywords: Ziziphus jujuba - colon cancer - azoxymethane - blood cells - aberrant crypt foci

Asian Pac J Cancer Prev, 16 (17), 7561-7566

\section{Introduction}

Cancer is the most important public health burden around the globe. Colon cancer is one of the most common malignancies in many parts of the world (Abdull Razis and Noor, 2013). Colorectal cancer (CRC) is the third most commonly diagnosed cancer and the third leading cause of cancer related death in the world. The incidence of CRC in the developing countries is rapidly increasing, due to alteration in life style (Siegel et al., 2013). It is one of the leading causes of cancer death worldwide and is the third most common form of malignancy in both men and women (Hamiza et al., 2012). It is also one of the fastest emerging gastrointestinal cancers in the Asia Pacific region (Goh et al., 2005). In China the incidence rate has been on the rise; from 2003 to 2007 , it was 28.08 per 100,000 males and ranked third among all cancer cases (Chen et al., 2012; Song et al., 2015). Colon cancer is the third cause of cancer overall and the second leading cause of cancer-related death in Europe and the United States. Approximately $50 \%$ of colorectal cancer patients develop metastases; prognosis for these patients is poor, with 5-year survival rates of $<10 \%$ (Absenger et al., 2013). CRC ranks the third place of cancer overall and the second leading cause of cancer-related death affecting both males and females in Europe (Siegel et al., 2013; Stotz et al., 2014).

In Taiwan, the incidence of CRC has increased in recent years due to Western lifestyle. Newly diagnosed CRC cases were the highest in number among all malignancies. In 2007, there were 10,511 newly diagnosed CRC patients, and 4470 persons died of CRC in the same year (BHP, 2007, Chiang et al., 2012). CRC is one of the leading causes of cancer death in Taiwan. Among all cases, about $70-80 \%$ of patients were diagnosed with stage I to III diseases (BHP, 2007). Colon cancer ranks number 2 in top 10 cancer incidence and mortality rates per 100,000 in 2011, with 14,087 cases and incidence rate of $60.65 \%$ according to Taiwan cancer registry (TCR, 2011). The consumption of diet-containing carcinogens is associated with increasing risk of colon cancer and it was also documented that high consumption of plant-based foods might decrease the cancer risk (Abdull Razis and Noor, 2013). Epidemiological studies have indicated that CRC is strongly related with diet, and thus it may be possible to prevent the incidence of this cancer through dietary modification (Pandurangan and Esa, 2014). 
Ziziphus jujuba (ZJ), a traditional Chinese herb belonging to the genus Zizyphus (Rhamnaceae), is mainly distributed in the subtropical regions of Asia such as China and Taiwan (Li et al., 2007a, b). Its fruits have long been used as crude drugs in traditional Chinese medicine to purify the blood and aid digestion. The sarcocarp of ZJ are rich in polysaccharides (Sun et al., 2011), have a delicate taste and red in color. Therefore, the sarcocarp of $\mathrm{ZJ}$ are exploited in Chinese beverages and functional foods (Yue et al., 2014). ZJ fruit is commonly used as a functional food against various diseases including diabetes, digestive disorders, diarrhea, skin infections, liver and urinary complications (Li et al., 2007a, b). The increasing demand for $\mathrm{ZJ}$ in food and pharmaceutical industries, its production has increased rapidly in the last decade, and $90 \%$ of the worldwide production was consumed by Chinese ( $\mathrm{Li}$ et al., 2007b). The major nutrients in ZJ include carbohydrate (80.86- 85.63\%), protein $(4.75-6.86 \%)$, lipid (0.37$1.02 \%)$, moisture (17.38-22.52\%), and ash (2.26-3.01) (Li et al., 2007a). However, little attention has been focused on the biological functions of ZJ sarcocarp (Yue et al., 2014). In the present study, we aimed to investigate the effect of dietary ZJ against colitis-associated colon cancer. We therefore hypothesize that dietary ZJ would attenuate the formation of ACF, circulating leucocyte and delay the progression of colon cancer.

\section{Materials and Methods}

\section{Chemicals}

Azoxymethane was purchased from Sigma-Aldrich chemical company, St. Louis, USA. Dextran sulfate sodium (MW ca 40,000) was purchased from Alfa Aesar, UK. All other chemicals and reagents used were of analytical grade. ZJ was purchased from Gong-guan Farmer's Cooperative, Miaoli, Taiwan.

\section{Animals}

Male C57BL/6J mice 6-7 weeks old and weighing 15-20 g was purchased from our institutions Laboratory Animal Center. They were given pellet diet and water ad libitum. They had a 12-h light/dark cycle and central air conditioning $\left(25^{\circ} \mathrm{C}, 70 \%\right.$ humidity $)$ throughout the experiment. The animal care and experimental protocols were in accordance with nationally approved guidelines (No. 99054).

\section{Experimental design}

After 1 week of acclimation, the mice were randomly assorted into five different experimental groups. Group N $(n=6)$ was on regular diet and drinking water. Group C, group CJ5, and group CJ10 ( $\mathrm{n}=8 \sim 12$, each group) were administered a single dose of AOM (10 mg/kg b.wt., i.p.). Starting 1 week after the injection, $2 \%$ DSS in the drinking water was administered to the mice in groups C, CJ5, and CJ10 for 7 and 14 days, followed by normal drinking water for the recovery period. A total of three cycles of $2 \%$ DSS were performed. Group CJ5 was fed with a $5 \% \mathrm{ZJ}$ fruit in diet and group CJ10 and group J10 $(n=6)$ were fed with a $10 \% \mathrm{ZJ}$ fruit in diet during the study period. The schematic experimental design was represented in Figure 1.
The mice were sacrificed on day 62 , and the colons, from the ileocecal junction to the anal verge, were removed. The colons were cut open longitudinally along the main axis, washed with phosphate-buffered saline $(\mathrm{pH}$ 7.4) and one part was stored for biochemical assays and one part was used for histology. The colons were prepared as "Swiss rolls," fixed in $10 \%$ formalin, and then paraffinembedded. The sections were stained with hematoxylin and eosin and then sections photographed.

\section{Assessment of colitis-associated colorectal aberrant crypt foci (ACF) grading}

Grading of ACF was done using histopathological sections, colon tissue from experimental mice were placed in $10 \%$ formalin. The tissues were dehydrated using a graded percentage of ethanol and then fixed in paraffin wax for $1 \mathrm{~h}$ to form blocks. The blocks were trimmed and cut into $4 \mu \mathrm{m}$ thick section, stained with hematoxylin and eosin (H\&E), and then mounted sections using Depex-Polystyrene dissolved in xylene mountant. The sections of colon tissue were examined under microscope (magnification, 100X) to assess ACF. ACF was classified into three categories including (a) hyperplastic ACF (elongated, no dysplasia), (b) low-grade dysplastic ACF (elongated, slightly crowded and pseudostratified nuclei, well polarity, and normal or slightly decreased number of goblet cells), (c) high-grade dysplastic ACF with elongated, crowded and pseudostratified nuclei, markedly increased N/C ratio, and significantly decreased number of goblet cells (Tammasakchai et al., 2015).

\section{Blood chemistry}

Blood sample were collected from the interior vena cava under light carbon dioxide anesthesia. Blood was drawn by venipuncture into tube coated with EDTA $0.5 \mathrm{~mol} / \mathrm{L} \mathrm{kept}$ at room temperature; however the measurements should be done within $4 \mathrm{~h}$ as per the instruments protocol. Measurements were done using Scil Vet Focus 5 Hematology Analyzer (Scil Animal Care Company, Gurnee, IL, USA) using automatic mode; 0.2 $\mathrm{mL}$ blood sample was used for analysis.

\section{Statistical analysis}

All statistical analyses were done using SPSS 11.0.1 (SPSS Inc., Chicago, IL). Data are mean \pm SD. Differences in the measured variables between each group were assessed using Fisher's Least Significant Difference test. Significance was set at $P<0.05$.

\section{Results}

\section{Dietary ZJ attenuated dysplastic ACF}

ACF formation can be classified into three grades with hyperplasia, low grade dysplasia and high grade dysplasia. High grade dysplasia is a distinct indication of cancer growth and progression. Dose dependent significant $(P<0.05)$ increase in the hyperplasia was observed in $\mathrm{ZJ}$ supplemented group compared to colon cancer induced mice. Low grade dysplasia was significantly $(P<0.05)$ increased in CJ5 and CJ10 groups relative to colon cancer induced mice. However, no significant difference was 
found between CJ5 and CJ10 groups. Dose dependent significant $(P<0.05)$ decrease in the high grade dysplasia was observed in $\mathrm{ZJ}$ supplemented group relative to colon cancer induced mice (Figure $2 \mathrm{a}$ and $2 \mathrm{~b}$ ).

Dietary ZJ decreased white blood cell (WBC) and lymphocyte ( $L Y$ ) counts

WBC is one of the most important parts of immune system in cancer. Colon cancer induced mice showed significant $(P<0.05)$ increase in WBC and LY counts compared to normal mice. ZJ supplemented to colon cancer group CJ5 and CJ10 groups showed significant $(P<0.05)$ decrease in $\mathrm{WBC}$ and $\mathrm{LY}$ count relative to colon cancer induced mice. No significant change in WBC and LY counts between control and ZJ alone supplemented group (Figure $3 \mathrm{a}$ and $3 \mathrm{~b}$ ).

Dietary ZJ decreased neutrophil (NE) and monocyte (MO) counts

Tumor-associated neutrophils play a major role in cancer biology. NE is significant portion of the inflammatory cell infiltrate in many models of cancer. MO represents a reservoir of inflammatory cells that contribute to cancer progression. NE and MO counts significantly $(P<0.05)$ increased in colon cancer induced mice compared to normal mice. ZJ supplemented to colon cancer group CJ5 and CJ10 groups showed significant $(P<0.05)$ decrease in $\mathrm{NE}$ and $\mathrm{MO}$ counts relative to colon cancer induced mice. No significant change in MO counts between control and ZJ alone supplemented group (Figure $4 a$ and $4 b)$.

\section{Dietary ZJ decreased eosinophil (EO) and basophil (BA)} counts

EO has significant favorable impacts on prognosis of colorectal cancer. Colon cancer induced mice showed a significantly $(P<0.05)$ increased EO and BA counts compared to normal mice. Dietary ZJ significantly $(P<0.05)$ reduced $\mathrm{EO}$ and $\mathrm{BA}$ counts in colon cancer

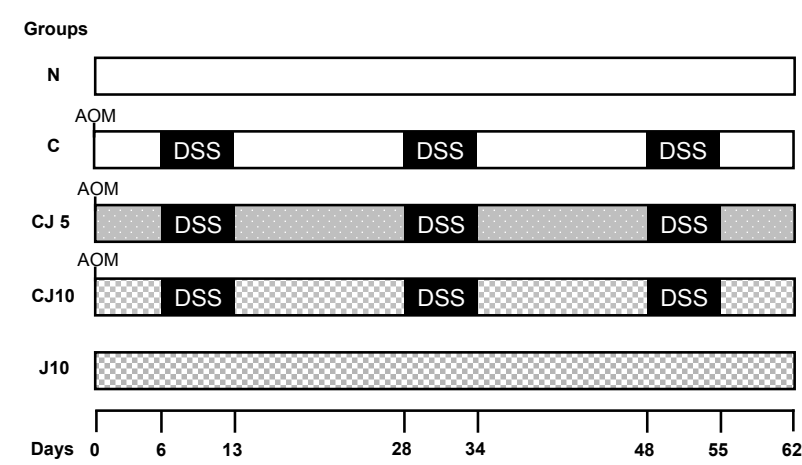

Figure 1. Experimental Protocol. Group N $(n=6)$ was on regular diet and drinking water. Group C, group CJ5, and group CJ10 ( $\mathrm{n}=8 \sim 12$, each group) were administered a single dose of AOM (10 mg/kg b.wt., i.p.). Starting 1 week after the injection, 2\% DSS in the drinking water was administered to the mice in groups $\mathrm{C}, \mathrm{CJ} 5$, and $\mathrm{CJ} 10$ for 7 and 14 days, followed by normal drinking water for the recovery period. A total of three cycles of $2 \%$ DSS were performed. Group CJ5 was fed with a $5 \% \mathrm{ZJ}$ fruit in diet and group CJ10 and group J10 $(n=6)$ were fed with a $10 \% \mathrm{ZJ}$ fruit in diet during the study period induced group CJ5 and CJ10 compared to colon cancer alone mice. No significant change in EO and BA counts between control and ZJ alone supplemented group (Figure $5 a$ and $5 b)$.

\section{Dietary ZJ decreased platelet (PLT) counts}

PLT is involved in the development of malignant tumors; a relationship between elevated platelet counts (thrombocytosis) and malignant tumors is well established. PLT counts significantly $(P<0.05)$ increased in colon cancer-induced mice compared to normal mice. $\mathrm{ZJ}$ supplemented to colon cancer group CJ5 and CJ10 showed

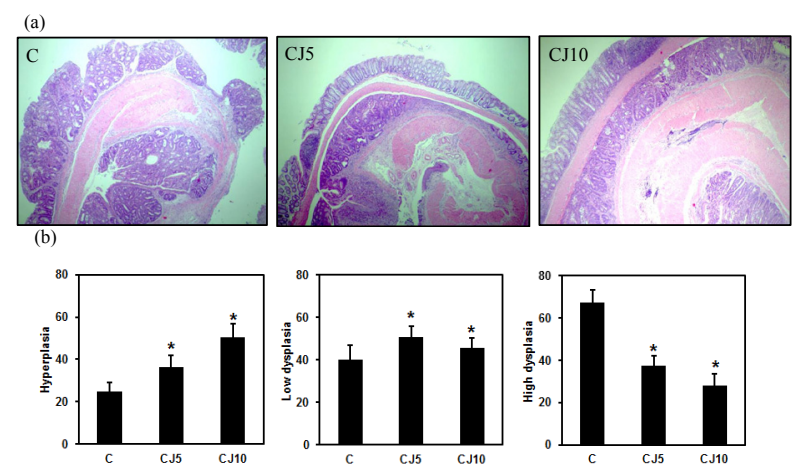

Figure 2. Dietary Influence of ZJ Fruit on ACF Formation in Colitis-associated Colon Cancer Mice. (See groups and treatment details in Figure 1 legend). Data are means \pm SD. Statically significant at $(P<0.05)$. \# - normal $(\mathrm{N})$ vs. colon cancer $(\mathrm{C})$; * - colon cancer $(\mathrm{C})$ vs. colon cancer $+\mathrm{ZJ}$ $5 \%(\mathrm{CJ} 5)$ and colon cancer $+\mathrm{ZJ} 10 \%(\mathrm{CJ} 10)$

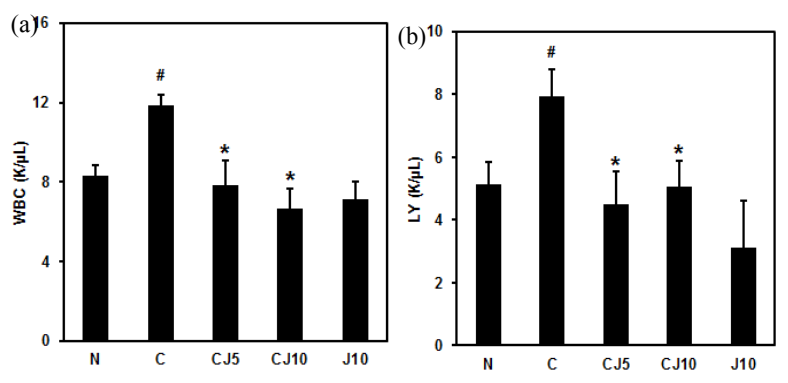

Figure 3. Dietary Influence of ZJ Fruit on WBC and LY in Colitis-Associated Colon Cancer Mice. (See groups and treatment details in Figure 1 legend). Data are means \pm SD. Statically significant at $(P<0.05)$. \# - normal $(\mathrm{N})$ vs. colon cancer (C); * - colon cancer (C) vs. colon cancer + ZJ 5\% (CJ5) and colon cancer + ZJ $10 \%$ (CJ10)
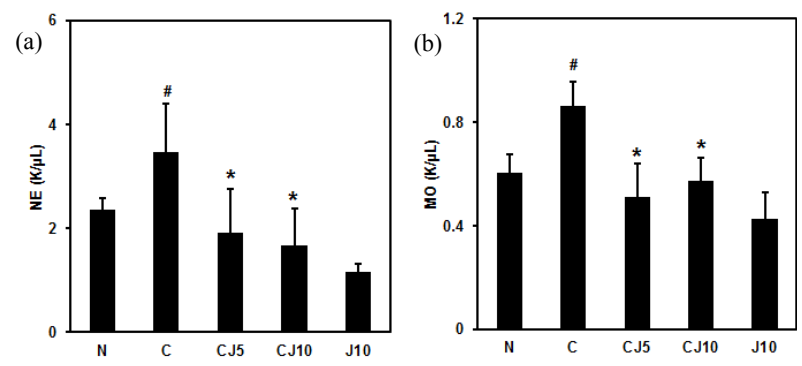

Figure 4. Dietary influence of $\mathrm{ZJ}$ fruit on NE and MO in Colitis-associated Colon Cancer Mice (See groups and treatment details in Figure 1 legend). Data are means \pm SD. Statically significant at $(P<0.05)$. \# - normal $(\mathrm{N})$ vs. colon cancer $(\mathrm{C})$; * - colon cancer $(\mathrm{C})$ vs colon cancer + ZJ $5 \%(\mathrm{CJ} 5)$ and colon cancer $+\mathrm{ZJ} 10 \%(\mathrm{CJ} 10)$ 

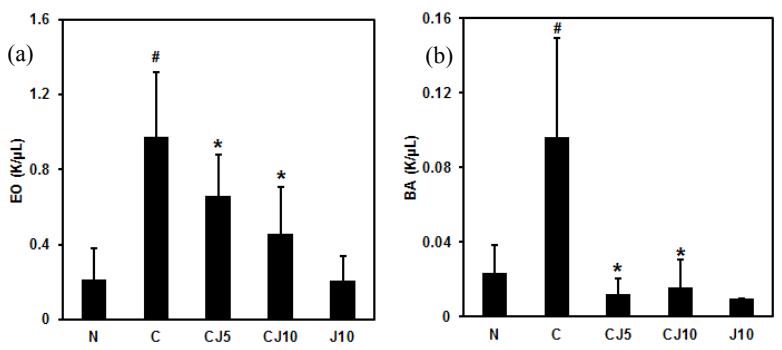

Figure 5. Dietary Influence of ZJ fruit on EO and BA in Colitis-Associated Colon Cancer Mice (See groups and treatment details in Figure 1 legend). Data are means \pm SD. Statically significant at $(P<0.05)$. \# - normal $(\mathrm{N})$ vs. colon cancer $(\mathrm{C}) ;{ }^{*}$ - colon cancer $(\mathrm{C})$ vs. colon cancer $+\mathrm{ZJ} 5 \%$ (CJ5) and colon cancer + ZJ 10\% (CJ10)

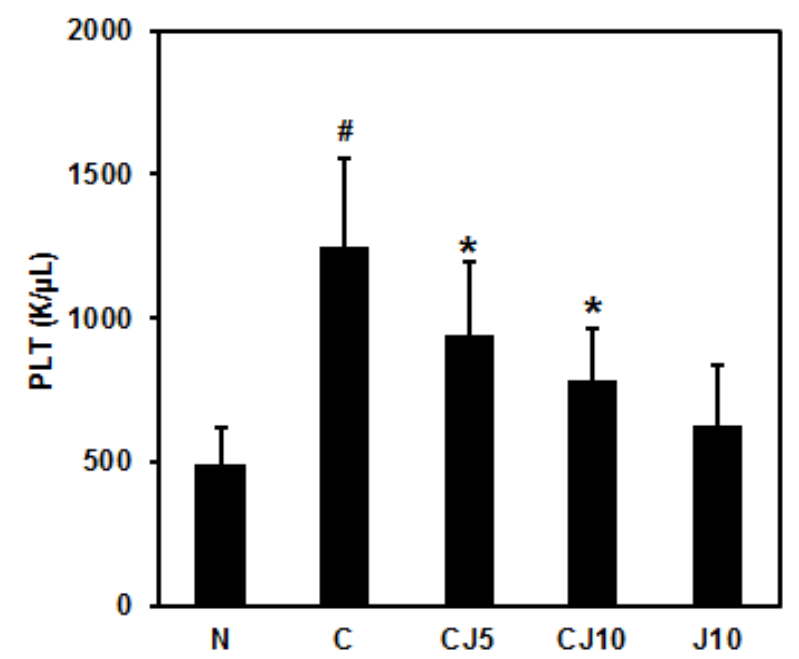

Figure 6. Dietary influence of ZJ fruit on PLT in Colitis-Associated Colon Cancer Mice (See groups and treatment details in Figure 1 legend). Data are means \pm SD. Statically significant at $(P<0.05)$. \# - normal $(\mathrm{N})$ vs. colon cancer $(\mathrm{C}) ;{ }^{*}$ - colon cancer $(\mathrm{C})$ vs. colon cancer + ZJ 5\% (CJ5) and colon cancer $+\mathrm{ZJ} 10 \%(\mathrm{CJ} 10)$

significant $(P<0.05)$ decrease in PLT counts relative to colon cancer induced mice. ZJ alone supplemented group of mice showed no significant change in PLT counts compared to control (Figure 6).

\section{Discussion}

Dietary supplementation of ZJ 5 and 10\% significantly decreased the colon cancer progression. Histopathological ACF evaluation showed decreased dysplasia in ZJ supplemented mice. WBC, LY, NE, MO, EO, BA, and PLT counts were decreased in ZJ supplemented colon cancer mice. Thereby, dietary ZJ supplementation delayed the progression of colon cancer from hyperplasia to dysplasia and ultimately adenocarcinoma and cancer.

$\mathrm{ZJ}$ delayed the progression of colon cancer by preventing the formation of $\mathrm{ACF}$, thereby attenuating the sequential process of hyperplasia, dysplasia, adenocarcinoma and cancer. AOM is a specific colon carcinogenic agent that induces the development of ACF in animal models (Guizani et al., 2013). ACF is considered to be precancerous lesions in the colorectum of humans and rodents. The number of crypts/foci has been shown to increase with time following carcinogenic AOM-treatment, and ACF demonstrate increased colonic cells proliferation (Guizani et al., 2013). The etiology of colon cancer is complicated and includes both genetic and environmental factors among the environmental factors; the dietary habits play a major role (Rajamanickam and Agarwal, 2008). Low intake of fibers, fruits and vegetables; and high intake of fat have been related with increased risk of colon cancer. It has been estimated that $30-40 \%$ of all tumors can be prevented with a correct lifestyle and diet, in particular colon cancer (Rajamanickam and Agarwal, 2008). The protective effects of plant-based foods and their byproducts have been attributed to their high amounts of phytonutrients that act as natural antioxidants against oxidative stress-mediated chronic disease, including cancer (Waly et al., 2015). Fruits concentrates protect against oxidative stress-mediated human diseases; including cancer (Waly et al., 2012). Fruits and vegetable intake are not strongly associated with colon cancer risk overall but may be associated with a lower risk of distal colon cancer (Koushik et al., 2007). In addition, high consumption of fruits and vegetables is associated with a reduced risk of CRC, especially of colon cancer (van Duijnhoven et al., 2009). Therefore, consumption of ZJ fruits attenuated the formation of ACF and delayed the progression of colon cancer.

Dietary ZJ decreased circulating WBCs and LYs in colon cancer mice. The association between WBC and the mortality risk of cancer, an elevated WBC correlated with an increased mortality risk for all cancers (Erlinger et al., 2004). A positive association between WBC and the risk of colon cancer was reported. Elevated WBC increases the incidence risk and mortality of colon cancer in men and women. Elevated WBC is a predictor of the incidence risk and mortality of colon cancer. Therefore, inflammation increases the risk of colon cancer (Lee et al., 2006). ZJ decreased WBC counts thereby it decreased the incidence and attenuated the inflammation ultimately delaying the development and progression of hyperplasia to colon cancer.

ZJ decreased LY counts in colitis-associated colon cancer. LYs are involved in cytotoxic cell death and cytokine production, which inhibits proliferation and metastatic capacity of tumor cells by immune response against the tumor. In colorectal cancer patients, tumorinfiltrating LYs has been shown to be independent prognostic factors of survival in all clinical stages. Furthermore, lymphocytosis is found to be significantly associated with increased overall survival in metastatic colorectal cancer patients (Absenger et al., 2013). ZJ decreased LY counts thus inhibited the proliferation and metastatic capacity of colon tumor.

Dietary ZJ decreased circulating NEs and MOs in colon cancer mice. Tumor-related MOs, are the main regulators of cancer inflammation, have an essential role in systemic inflammatory response to tumorous disease (Mantovani et al., 2008; Stotz et al., 2014). Tumorinfiltrating NEs and MOs have a crucial role in tumor development and progression (Mantovani et al., 2008). Macrophages are more differentiated MOs; develop from 
cells of the mononuclear phagocytic lineage that show specific phenotypic characteristics. The role of MOs in cancer development and progression is controversial, as inhibiting as well as enhancing potential of MOs in human cancer (Mytar et al., 2008; Stotz et al., 2014). Dietary ZJ decreased circulating NEs and MOs by attenuating inflammation and inhibiting colon tumor development and progression.

ZJ decreased EO and PLT counts in colitis-associated colon cancer. Thrombocytosis has been found to be associated with tumor metastasis and prognosis in malignant tumors including CRC. Elevated PLT might play some role in the progress of CRC and preoperative PLT counts might be a prognostic indicator in the CRC patients (Lin et al., 2012; Baranyai et al., 2013). Inflammation is responsible for increased mean PLT volume. Chronic inflammation increases the risk of developing colon cancer. The patients with colon cancer have higher mean platelet volume levels compared to the controls. Tumor cells express tissue factor and secrete cytokines that contribute to a prothrombotic microenvironment, which involves activation of PLTs. Activated PLTs may contribute to angiogenesis (Li et al., 2014). High blood and tissue EO counts to be associated with better CRC prognosis (Legrand et al., 2010). ZJ decreased EO and PLT counts thus inhibited the tumor metastasis and progression of colon tumor.

The implication of the present study is that dietary $\mathrm{ZJ}$ has the potential to attenuate ACF formation, inflammation through WBCs along with LYs and delayed the progression of colon cancer from hyperplasia to dysplasia and ultimately adenocarcinoma and cancer. Treatment for colon cancer is currently dictated by stage. Curative surgery with resection of macroscopic and microscopic evidence of the tumor is the primary treatment with adjuvant therapy (Hassan et al., 2015). Therefore, need for non-toxic therapeutic agents that are easy and effective to use for cancer prevention and treatment is essential. About $80 \%$ of all anticancer drugs are composed from natural products (Zhang et al., 2012; Dehghan Esmatabadi et al., 2015). ZJ might be a pivotal candidate for the dietary intervention against colon cancer. ZJ crude extracts isolated using different solvents was shown to be effective against hepatoma by inducing apoptosis and cell cycle arrest at the G2/M phase (Huang et al., 2007; Hung et al., 2012). PHY906, is a decoction of a mixture of four herbs, one of it is ZJ Mill. Preclinical studies have shown that PHY906 enhances the therapeutic indices of a broad spectrum of anticancer agents (Liu et al., 2012). In the present study ZJ alone supplemented group showed mild decrease in circulating LY, NE, MO and BA. Therefore, ZJ might possess anti-inflammatory and immuno-modulatory functions. However, further studies are needed to prove this fact. In addition present study showed the evidence that inclusion of ZJ 5 and $10 \%$ in food can attenuate the progression of colon cancer from hyperplasia to dysplasia and ultimately adenocarcinoma and cancer. In addition, it decreased circulating tumor-related leucocytes, main regulators of cancer inflammation. Therefore, dietary consumption of $\mathrm{ZJ}$ fruit might attenuate the formation of $\mathrm{ACF}$ and delayed the progression of colon cancer.

\section{Acknowledgements}

This study was supported by the Taiwan Ministry of Sciences and Technology [Grant 102-2314-B-006-028MY2].

\section{References}

Abdull Razis AF, Noor NM (2013). Cruciferous vegetables: dietary phytochemicals for cancer prevention. Asian Pac J Cancer Prev, 14, 1565-70.

Absenger G, Szkandera J, Pichler M, et al (2013). A derived neutrophil to lymphocyte ratio predicts clinical outcome in stage II and III colon cancer patients. Br J Cancer, 109, 395-400.

Baranyai Z, Jósa V, Krzystanek M, et al (2013). Evaluation of thrombocytosis as predictive factor in colorectal cancer. Magy Seb, 66, 331-7.

BHP, Bureau of Health Promotion (2007). Department of Health, R.O.C. (Taiwan). http://www.bhp.doh.gov.tw/BHPnet/ Portal/StatisticsShow. aspx?No0200911300001

Chen Q, Liu ZC, Cheng LP, et al (2012). Analysis of incidence and mortality of colorectal cancer in china 2003-2007. Chin Cancer, 21, 179-82.

Chiang SF, Hung HY, Tang R, et al (2012). Can neutrophil-tolymphocyte ratio predict the survival of colorectal cancer patients who have received curative surgery electively? Int J Colorectal Dis, 27, 1347-57.

Dehghan Esmatabadi MJ, Farhangi B, Safari Z, et al (2015). Dendrosomal curcumin inhibits metastatic potential of human SW480 colon cancer cells through Down-regulation of Claudin1, Zeb1 and Hef1-1 gene expression. Asian Pac $J$ Cancer Prev, 16, 2473-81.

Erlinger TP, Muntner $P$, Helzlsouer KJ (2004). WBC and the risk of cancer mortality in a national sample of U.S. adults: results from the Second National Health and Nutrition Examination Survey mortality study. Cancer Epidemiol Biomarkers Prev, 13, 1052-6

Goh KL, Quek KF, Yeo GT, et al (2005). Colorectal cancer in Asians: a demographic and anatomic survey in Malaysian patients undergoing colonoscopy. Aliment Pharmacol Ther, 22, 859-64.

Guizani N, Waly MI, Singh V, Rahman MS, et al (2013). Nabag (Zizyphus spina-christi) extract prevents aberrant crypt foci development in colons of azoxymethane-treated rats by abrogating oxidative stress and inducing apoptosis. Asian Pac J Cancer Prev, 14, 5031-5.

Hamiza OO, Rehman MU, Tahir M, et al (2012). Amelioration of 1,2 Dimethylhydrazine (DMH) induced colon oxidative stress, inflammation and tumor promotion response by tannic acid in Wistar rats. Asian Pac J Cancer Prev, 13, 4393-402.

Hassan AS, Naicker M, Yusof KH, Wan Ishak WZ (2015). Prognostic factors and the role of adjuvant chemotherapy in post-curative surgery for Dukes B and C colon cancers and survival outcomes: a Malaysian experience. Asian Pac J Cancer Prev, 16, 2237-43.

Huang X, Kojima-Yuasa A, Norikura T, et al (2007). Mechanism of the anti-cancer activity of Zizyphus jujuba in HepG2 cells. Am J Chin Med, 35, 517-32.

Hung CF, Hsu BY, Chang SC, Chen BH (2012). Antiproliferation of melanoma cells by polysaccharide isolated from Zizyphus jujuba. Nutrition, 28, 98-105.

Koushik A, Hunter DJ, Spiegelman D, et al (2007). Fruits, vegetables, and colon cancer risk in a pooled analysis of 14 cohort studies. J Natl Cancer Inst, 99, 1471-83.

Lee YJ, Lee HR, Nam CM, Hwang UK, Jee SH (2006). White blood cell count and the risk of colon cancer. Yonsei Med 


$$
J, 47,646-56 \text {. }
$$

Legrand F, Driss V, Delbeke M, et al (2010). Human eosinophils exert TNF- $\alpha$ and granzyme A-mediated tumoricidal activity toward colon carcinoma cells. J Immunol, 185, 7443-51.

Li JW, Ding SD, Ding XL (2007a). Optimization of the ultrasonically assisted extraction of polysaccharides from Zizyphus jujuba cv. Jinsixiaozao. J Food Eng, 80, 176-83.

Li JW, Chen YY, Ding SD, Zhang LF (2007b). Isolation and analysis of a novel proteoglycan from Zizyphus jujuba cv. Jinsixiaozao. J Food Drug Anal, 15, 271-7.

Li JY, Li Y, Jiang Z, Wang RT, Wang XS (2014). Elevated mean platelet volume is associated with presence of colon cancer. Asian Pac J Cancer Prev, 15, 10501-4.

Lin MS, Huang JX, Zhu J, Shen HZ (2012). Elevation of platelet count in patients with colorectal cancer predicts tendency to metastases and poor prognosis. Hepatogastroenterol, 59, 1687-90.

Liu SH, Cheng YC (2012). Old formula, new Rx: the journey of PHY906 as cancer adjuvant therapy. J Ethnopharmacol, 140, 614-23.

Mantovani A, Allavena $P$, Sica A, Balkwill F (2008). Cancerrelated inflammation. Nature, 454, 436-44.

Mytar B, Baj-Krzyworzeka M, Majka M, Stankiewicz D, Zembala M (2008). Human monocytes both enhance and inhibit the growth of human pancreatic cancer in SCID mice. Anticancer Res, 28, 187-92.

Pandurangan AK, Esa NM (2014). Luteolin, a bioflavonoid inhibits colorectal cancer through modulation of multiple signaling pathways: a review. Asian Pac J Cancer Prev, 15, 5501-8.

Rajamanickam S, Agarwal R (2008). Natural products and colon cancer: current status and future prospects. Drug Devel Res, 69, 460-71.

Siegel R, Naishadham D, Jemal A (2013). Cancer statistics, 2013. CA Cancer J Clin, 63, 11-30.

Song Y, Liu M, Yang F, et al (2015). Dietary fibre and the risk of colorectal cancer: a case- control study. Asian Pac J Cancer Prev, 16, 3747-52.

Stotz M, M Pichler, G Absenger, et al (2014). The preoperative lymphocyte to monocyte ratio predicts clinical outcome in patients with stage III colon cancer. Brit J Cancer, 110, 435-40.

Sun YF, Liang ZS, Shan CJ, Viernstein H, Unger F (2011). Comprehensive evaluation of natural antioxidants and antioxidant potentials in Ziziphus jujuba Mill. var. spinosa (Bunge) Hu ex H.F. Chou fruits based on geographical origin by TOPSIS method. Food Chem, 124, 1612-9.

Tammasakchai A, Chaiyasut C, Riengrojpitak S, Suwannalert $P$ (2015). Unpolished thai rice prevents ACF formation and dysplastic progression in aom-induced rats and induces apoptosis through redox alteration in $\mathrm{CaCo}-2$ cells. Asian Pac J Cancer Prev, 16, 2827-32.

TCR, Taiwan Cancer Registry (2011). http://tcr.cph.ntu.edu.tw/ main.php?Page=N2

Van Duijnhoven FJ, Bueno-De-Mesquita HB, Ferrari $P$, et al (2009). Fruit, vegetables, and colorectal cancer risk: the European Prospective Investigation into Cancer and Nutrition. Am J Clin Nutr, 89, 1441-52.

Waly MI, Al-Ghafri BR, Guizani N, Rahman MS (2015). Phytonutrient Effects of Date Pit Extract against Azoxymethane- Induced Oxidative Stress in the Rat Colon. Asian Pac J Cancer Prev, 16, 3473-7.

Yue Y, Wu S, Zhang H, et al (2014). Characterization and hepatoprotective effect of polysaccharides from Ziziphus jujuba Mill. var. spinosa (Bunge) Hu ex H. F. Chou sarcocarp. Food Chem Toxicol, 74, 76-84.

Zhang Z-Y, Dong J-H, Chen Y-W, et al (2012). Galectin-9 acts as a prognostic factor with antimetastatic potential in hepatocellular carcinoma. Asian Pac J Cancer Prev, 13, 2503-9. 